\title{
Bacterial pyomyositis in a patient with aplastic anaemia
}

\author{
Ronald Mitsuyasu \\ M.D.
}

\author{
Robert Peter Gale \\ M.D., Ph.D.
}

\begin{abstract}
UCLA Bone Marrow Transplant Unit, UCLA School of Medicine, The Center for the Health Sciences, Los Angeles, CA 90024
\end{abstract}

\begin{abstract}
Summary
Bacterial pyomyositis is common in the tropics but is rare in temperate climates. A patient with aplastic anaemia who had never left the continental United States developed bacterial pyomyositis secondary to Staphylococcus aureus which responded to antibiotics and surgical drainage. Bacterial pyomyositis should be considered in the differential diagnosis of fever and myalgias in the immunocompromised patient.
\end{abstract}

\section{Introduction}

Bacterial pyomyositis is a common disease in the tropics accounting for $1-2 \%$ of surgical hospital admissions in some areas (Horn and Masters, 1968; Foster, 1965). It has been reported from individuals recently returned from the tropics (Levin, Gardner, and Waldvogel, 1971; Penman and Rothwell, 1968) but only rarely in individuals who have never travelled abroad (Rogers, 1973; Altrocchi, 1971; Echeverria and Vaughn, 1975; Williams and Thomas, 1975). A case has been observed of bacterial pyomyositis in a patient with aplastic anaemia who had never left the United States.

\section{Case report}

A 30-year-old Mexican-American male with aplastic anaemia was referred to UCLA* Hospital. Six months before admission he had developed progressive fatigue, weakness, palpitations and petechiae over the lower extremities. A diagnosis of aplastic anaemia was made based on typical haematological findings and bone marrow examination. One month before admission the patient had developed fever and diffuse myalgias. He was admitted to another hospital, with a fever of $40^{\circ} \mathrm{C}$, petechiae, and haematomata and diffuse muscle tenderness most severe over the calves. All cultures including blood were negative save for a urine culture which grew $>10^{5}$ colonies of Staphylococcus aureus. Cephalothin and gentamicin were started empirically and the

* University of California, Los Angeles.

Requests for reprints to Dr R. P. Gale. fever rapidly subsided. Examination at the time of transfer to UCLA revealed petechiae and multiple ecchymoses over the calves, thighs, and forearms and diffuse soft tissue swelling of all extremities. There was a $3-\mathrm{cm}$ tender, erythematous, slightly fluctuant subcutaneous nodule on the dorsum of the left hand and a $2-\mathrm{cm}$ non-fluctuant lesion on the ventral aspect of the left wrist. The remainder of the physical examination was unremarkable. There were no cardiac murmurs. The liver and spleen were not enlarged and there was no lymphadenopathy. The $\mathrm{Hb}$ was $10.4 \mathrm{~g} / \mathrm{dl}$ and the WBC $0.6 \times 10^{\circ} / 1$ with $5 \%$ neutrophils, $90 \%$ lymphocytes and $5 \%$ monocytes. No eosinophils were observed. Platelets were $13 \times$ $10^{9} / 1$. Muscle enzymes including CPK, LDH and aldolase were normal. Liver function tests indicated an SGOT and SGPT of 262 and 879 i.u./1 respectively. Urinanalysis was normal. Cultures of urine, blood, throat, and stool were negative. $\mathrm{HB}_{\mathrm{s}} \mathrm{Ag}$ was not detected. Complement fixation titre for CMV was positive at $1: 64$. A Gram stain of material from one lesion revealed many Gram-positive cocci in clusters, and cultures grew a $\beta$-lactam-resistant $S$. aureus. Anaerobic cultures and cultures for Mycobacterium tuberculosis were negative. Several other lesions were surgically incised and drained and all cultures grew $S$. aureus. The patient was treated with intravenous oxacillin to which he responded promptly with a defervescence of fever and a healing of the abscesses. He had never travelled outside the United States and denied any history of muscle trauma, intramuscular injections, or parasitic infection.

\section{Discussion}

This patient presented with typical features of bacterial pyomyositis. In most cases, the onset is subacute with ill defined muscle pain preceding fever by several days. Large muscle groups, such as the quadriceps and spinal trunk muscles, are commonly involved (Fett, 1973). The lesions are generally hard or elastic but erythema and fluctuation may occur. Muscle wasting is rare and muscle enzymes are generally normal. The WBC count and 
ESR are frequently elevated (Jordan et al., 1976; Taylor et al., 1973). Eosinophilia is variably present and may be related to parasitic infection in some instances. $S$. aureus is the most frequent pathogen but S. epidermidis, Streptococcus spp., and Pasteurella spp. have been reported (Foster, 1965; Svane, 1971; Barrett and Gresham, 1958).

The pathogenesis of pyomyositis is unknown. Skeletal muscle is relatively resistant to bacterial infection, and muscle abscesses occur only rarely in patients with bacterial sepsis (Levin et al., 1971; Smith and Vickers, 1960). Halsted (1924) was unable to produce abscesses in dogs with direct intramuscular injection of Staph. aureus even when local blood supply was severely compromised. Other factors such as parasitic and viral infection, haematomas, ascorbic acid deficiency, and general malnutrition may predispose to pyomyositis (Ashken and Cotton, 1963; Levin et al., 1971; Taylor et al., 1973; Tilma, 1977; O'Brien, 1974). Anand and Evans (1964) identified Dracunculus medinensis in 8 patients with intermuscular abscesses, and O'Brien (1974) was able to demonstrate primary nematode infection in several cases of intramuscular abscesses with secondary staphylococcal infection. Filariasis has been reported in association with pyomyositis, but this may have been coincidental. Electron micrographs have revealed virus-like particles in muscle tissue from some patients with pyomyositis but most viral serological and culture studies have been negative (Taylor, Fluck and Fluck, 1976). Joseph (1975) has proposed a pathogenic mechanism in which bacteraemia may produce abscesses in tissue damaged by trauma or infection. While this is an attractive hypothesis, pyomyositis develops only rarely in conditions of altered host defences that are associated with staphylococcal bacteraemia. Jordan et al. (1976), have reported staphylococcal myositis in a patient with acute myelomonocytic leukaemia and leucopenia secondary to chemotherapy. In the present case, severe aplastic anaemia was a likely disposing factor. Thrombocytopenia with intramuscular haemorrhage and local tissue damage may have been contributory.

The patient developed bacterial pyomyositis with clinical features of 'tropical' pyomyositis. Prompt antimicrobial therapy and surgical drainage resulted in complete resolution of the infection. Bacterial pyomyositis should be suspected in immunocompromised patients with fever and diffuse myalgias, even in the absence of localized signs of inflammation.

\section{Acknowledgments}

Supported by Grants CA-23175, CA-12800, CA-15688, and RR-865 from the National Institutes of Health and the U.S. Public Health Service.

Robert Peter Gale is a Scholar of the Leukemia Society of America.

\section{References}

AltrocChI, P.H. (1971) Spontaneous bacterial myositis. صి Journal of the American Medical Association, 217, 819.

Anand, S.V. \& Evans, K.T. (1964) Pyomyositis. British $\overrightarrow{0}$ Journal of Surgery, 51, 917.

AshkeN, M.H. \& CotTon, R.E. (1963) Tropical skeletal $\overrightarrow{\vec{\omega}}$ muscle abscesses (pyomyositis tropicans). British Journal of Surgery, 50, 846.

Barrett, A.M. \& Gresham, G.A. (1958) Acute streptococcal myositis. Lancet, i, 347.

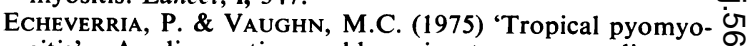
sitis'. A diagnostic problem in temperate climates. of American Journal of Diseases of Children, 129, 856.

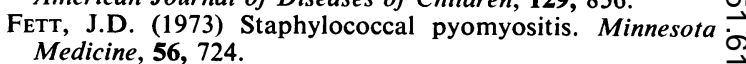
FOSTER, W.D. (1965) The bacteriology of tropical pyomyositis $\underset{\supset}{\mathrm{J}}$
in Uganda. Journal of Hygiene, 63, 517 .

Halsted, W.S. (1924) Surgical Papers. P. 103, Johns Hopkins $\vec{c}$ Press, Baltimore.

Horn, C.V. \& Masters, S. (1968) Pyomyositis tropicans in Uganda. East African Medical Journal, 45, 463.

JORDAN, G.W., BAUER, R., WONG, G.A. \& BARRY, A.L. (1976) Staphylococcal myositis in a compromised hos. $\overrightarrow{0}$ Successful treatment with the synergistic combinatie of nafcillin and gentamicin. Western Journal of Medicinæ 124, 140.

JoSEPH, S.C. (1975) Pyomyositis, a 'tropical' disease. Ame? ican Journal of Diseases of Children, 129, 775.

LeVIN, M.J., Gardner, P. \& WALDVoGel, F.A. (1971) 'Tropical' pyomyositis. New England Journal of Medicine, ฏે 284, 196.

O'Brien, D.D. (1974) Pyomyositis in London. British Medical Journal, 1, 78.

Penman, H.G. \& Rothwell, A.G. (1968) Tropical myositis. New Zealand Medical Journal, 68, 246.

Rogers, D.W. (1973) Case of pyomyositis occurring in London. British Medical Journal, 3, 679.

SMith, I.M. \& Vickers, A.B. (1960) Natural history of 338 흘 treated and untreated patients with staphylococcal bacter- $\exists$ aemia. Lancet, i, 1318.

Svane, S. (1971) Peracute spontaneous streptococcal myositis. A report on 2 fatal cases with review of literature. Acta chirurgica scandinavica, 137, 155.

Taylor, J.F., Shaw, B., Bluming, A., Briers, P., Friedman, E., Henderson, B., Horn, C., Mohan, S. \& Pike, M. (1973) Tropical myositis, clinical and laboratory studies. African Journal of Medical Sciences, 4, 409.

TÁYloR, J.F., FluCK, D. \& FluCK, D. (1976) Tropical 을 myositis: ultrastructural studies. Journal of Clinical $\mathrm{N}$ Pathology, 29, 1081.

Tilma, A. (1977) Pyomyositis in Swaziland. South African $N$ Medical Journal, 51, 39.

Williams, R.H.P. \& Thomas, P. (1975) An unusual case of $\mathfrak{\omega}$ spontaneous bacterial myositis. Postgraduate Medical Journal, 51, 255. 